\title{
Whole Exome Sequencing Identifies a Novel Pathogenic BMPR2 Variant in Pulmonary Atresia
}

\author{
Muyu Qi ${ }^{1}$, Xiaoping $\operatorname{Lan}^{1}$, Rufang Zhang ${ }^{1}$, Jia $\mathrm{Li}^{1}$, Junwen $\mathrm{Ge}^{1}$, and Li Shen ${ }^{1}$ \\ ${ }^{1}$ Shanghai Children's Hospital
}

November 13, 2020

\begin{abstract}
Objective Pulmonary atresia (PA) is a rare type of complex cyanotic congenital heart defect mainly characterized by an undeveloped pulmonary valve or pulmonary artery. herefore, defining a disease-causing gene mutation in a pulmonary atresia family becomes a possible method of the genetic counseling, future prenatal diagnosis, and therapeutic approaches of pulmonary atresia. Methods Blood samples of six members in a PA family were collected, and the genomic DNA was extracted using the QIAamp DNA Blood Mini Kit. The gene detection was performed using the second-generation sequencing gene Panel. Results Genetic testing results indicates as follows: A heterozygous mutation originated from maternal inheritance was detected in the BMPR2 gene of the proband's genomic DNA. The pathogenic gene was at c.2804C $>$ T (p. A935V). The mutation was also detected in the genomic DNA of the proband's elder brother(III-1), but not in other family members. Conclusion To our knowledge, this is the first study to report the BMPR2 variant responsible for pulmonary atresia. The frequency of c. $2804 \mathrm{C}>\mathrm{T}$ (p. A935V) mutation detected in this family is extremely low in the normal population (1/ 246048). The mutation was highly conservative in different species. And SIFT (sorting intorlerant from tolerant) predicts it to be a harmful mutation.
\end{abstract}

Whole Exome Sequencing Identifies a Novel Pathogenic BMPR2 Variant in Pulmonary Atresia MUYU QI, XIAOPING LAN, JIA LI, JUNWEN GE, LI SHEN, RUFANG ZHANG

All these authors are from Children's Hospital of Shanghai. Muyu Qi, Jia Li, Junwen Ge, Li Shen and Rufang Zhang are doctors of the department of Cardiac Surgery. Xiaoping Lan is researcher of Central Laboratory of Children's Hospital of Shanghai. The highest academic degree of Li Shen, RuFang Zhang and Xiaoping Lan are doctor, and other authors are masters.

Corresponding author: LI SHEN, Ph.D, Vice Director of Department of Cardiac Surgery, Children's Hospital of Shanghai, No. 355 of Luding Road, Putuo District, Shanghai, China, email:shenli@shchildren.com.cn,Tel: 18964647822

Co-corresponding author: Rufang Zhang, Professor, Director of Department of Cardiac Surgery, Children's Hospital of Shanghai, No. 355 of Luding Road, Putuo District, Shanghai, China, email:zhangrf@shchildren.com.cn, Tel: 18917128398

Key words: pulmonary atresia; gene mutation; BMPR2 gene

Running title: Whole Exome Sequencing Identifies a variant in PA

Separate word counts: 3118

Funding: Chinese National Natural Science Foundation, No. 81371499. The sponsor of this fund is also the corresponding author of this article. 


\section{Introduction:}

Congenital heart disease is the most common birth malformation. Pulmonary atresia is a rare type of complex cyanotic congenital heart defect mainly characterized by an undeveloped pulmonary valve or pulmonary artery, thus obstructing the blood travel from the heart to pick up oxygen from the lungs. The morbidity of the disease is $1.3 \%-3.4 \%$ of all congenital heart malformations with poor prognosis ${ }^{1}$. About $20 \%$ of children with pulmonary atresia with ventricular septal defect die within 2 years old ${ }^{2}$. During the fetal life, a development of prenatal ultrasound technology and a better understanding of progression of pulmonary atresia, become crucial points for the choice of lifetime of medical care including multiple surgeries or other interventions. However, ultrasound examination could not detect all potential children, especially those with major artery-pulmonary collateral arteries(MAPCAs) ${ }^{3}$. Under normal circumstances, patients do not purely suffer from PA. Usually the PA patients could combine with other heart diseases to survive. These heart diseases make it left to right shunts that make it possible for the blood to pick up oxygen from lungs through some natural passages among heart or their arteries, such as ventricular septum defect, patent ductus arteriosus and atrial septum defect. Otherwise the patients cannot survive. Accordingly, the PA is divided into two categories: pulmonary atresia with ventricular septal defect (PA/VSD) and pulmonary atresia with intact ventricular septum (PA/IVS). Among them, the ventricular septal integrity of the PA is also known as right heart dysplasia syndrome. Such patients often combine with the disappearance of the right ventricular outflow tract, resulting in a poor right ventricular development which leads to the loss of opportunities of biventricular repair ${ }^{4}$. Therefore children with PA often develop heart failure and cyanosis clinically. Presently PA is closely related to the heterozygous deletion of chromosome 22q11.2, and the range of the deletion was from $1437 \mathrm{Mbs}$ to $2706 \mathrm{Mbs}^{5}$. The ratio of deletion mutation among the patients is about $30 \%$ to $40 \%$. In children with PA with tetralogy of Fallot, the incidence of this heterozygous deletion is about $13 \%{ }^{6}$.If the child has major artery-pulmonary collateral arteries (MAPCAs), the incidence of heterozygous deletion can be as high as $50 \%^{7}$. At present, the main treatment of PA is still surgery. With the rise of 3D printing technology in recent years, personalized computer modeling of children with 3D printing technology helps to improve the accuracy of the surgical procedure, while mostly reducing intraoperative radiation and contrast load, and partly assists reduce the complications it causes ${ }^{8,9}$. Although the surgical technique and perioperative management have been improved, the prognosis of PA is still poor ${ }^{10}$. Therefore, screening for children with PA in the neonatal or even fetal period through genetic or biomarker methods will greatly increase the detection rate, so that children with PA gain early diagnosis and treatment.

At present, several genetic variants have been found to be associated with the pathogenesis of PA, including chromosome copy number variation and single gene mutation. Chromosomal variation mainly includes chromosome 22q11.2 heterozygous deletion, chromosome 1q21.1 heterozygous deletion, 16p13.1, 5q14.1, 5q14.1, 10p13 duplication and 17q13.2 chromosome deletion. And the related genes located in these CNVs includes the MYH11, ABCC6, NDE1, TBX1, DHFR, PDE88, AP3B, ARSB,DMGDH, CUBN,CAMTA2, CHRNE, GP18A, ENO,GJA5, GJA8, BCL9, etc ${ }^{5,11}$. These genes were reported to be related to the metabolism in folate and vitamin $\mathrm{B} 12^{12,13}$. At the same time, several single gene mutation sites were also found, including GJA5, GJA1, GDF1, MTHFR and etc ${ }^{1}$. Bone morphogenetic protein type 2 receptor (BMPR2) is one of the transforming growth factor- $\beta$ superfamily receptors, which performs diverse roles in various tissues and organs, including pulmonary vascular endothelium, pulmonary vascular smooth muscle, vasculogenesis, and osteogenesis. So far, the BMPR2 and its corresponding type 1 receptors both function in the development or differentiation of the embryos, organs and bones ${ }^{14}$. The BMPR2 consists of 1,038 amino acids, and functions within various fields such as extracellular, transmembrane, kinase, and C-terminal cytoplasmic domains ${ }^{15}$. Reports presented that the BMPR2 enhances almost every step of vascular development. Variations of the BMPR2 gene leads to various construcions and fuctions of the gene, and these changes are particularly associatied with clinical disorders including pulmonary arterial hypertension (PAH), cancers, obesity, metabolic diseases and so on ${ }^{15-19}$. BMPR2 plays important roles in various biological pathways, and to promote the vascular development, canonical BMPR2-mediated signaling cascade is reported to be associated with human microvascular endothelial cells (HMVECs), human umbilical vein endothelial cells (HUVECs), and aortic endothelial cells ${ }^{20}$. 
In the present study, we collected a PA family with two affected and four unaffected members. To uncover the novel pathogenic genes or variants, we performed whole exome sequencing on six family members. With bioinformatics tools, we identified a novel variant at c.2804C $>$ T (p. A935V) in BMPR2 as the diseasecausing variant, which may function in the heart development. To our knowledge, this is the first study to report the BMPR2 as a disease-causing variant for heritable PA.

\section{Data and methods}

\section{1 clinical features of the PA cases}

This study collected the PA family from Anhui Province, China, which was admitted to the Department of Cardiothoracic Surgery, Shanghai Children's Hospital. The family had 3 generations and 2 typical patients. The proband (III-2) was a male infant weighing $3600 \mathrm{~g}$ borned masure without any specific information about the disease on fetal screening echocardioraphy. He was soon found symptoms of cyanosis of face and mouth after birth and therefore admitted to the local hospital. Since the patient received an anti-infective treatment after which his symptoms were not relieved, he was referred to our hospital. The physical examination showed cyanosis, wheezing, and shortness of breath, and continuous murmurs could be caught on the precordium. His percutaneous arterial oxygen saturation (SpO2) level in the right arm, measured using pulse oximetry, decreased to below $70 \%$ without oxygen uptake. And the blood routine suggested WBC $20.25^{*} 10^{\wedge} 9 / \mathrm{L}$ (normal level: $8.00-12.00^{*} 10^{\wedge} 9 / \mathrm{L}$ ). Blood gas analysis indicated a CO2 retention and low oxygen saturation. The echocardiogram also showed a pulmonary atresia with intact ventricular septum and patent ductus arteriosus. He was started on prostaglandin immediately after admitted for ductal-dependent pulmonary blood flow. Inquired about the medical history, the proband had a history of repeated upper respiratory tract infections and pneumonia. The proband was finally diagnosed as pulmonary atresia with intact ventricular septum, patent ductus arteriosus and atrial septal defect, which showed typical symptoms of this disease. He went to the operating room for three times after which he did well with appropriate oxygen saturations.

Further family history research revealed that the proband's elder brother (III- 1) had the similar symptoms (Figure 1 ). The proband's elder brother was diagnosed as pulmonary atresia with ventricular septum defect based on the present history, relevant examination as well as physical examination. And he was also cured by operation. With whole exome sequencing, we found the proband's mother carries the mutant gene and had also presents related symptoms in our hospital. She presents an obesity (BMI=26.03), exhaustion after mild exercises or movements. It has been reported that mutation in BMPR2 could be associated with obesity, as well as several cardiovascular related diseases, all of which mainly present an exhaustion after movements.

\subsection{Methods}

\subsubsection{Diagnostic criteria of PA patients with cardiac color Doppler}

According to the latest clinical guidelines, prenatal PA was mainly diagnosed with four-dimensional color Doppler ultrasound, presenting an abnormal performance. And PA could be diagnosed early in the second phase of pregnancy (18-22 weeks) with this method. Such fetal four-dimensional color Doppler ultrasound abnormalities mainly exist in the pulmonary artery, tricuspid valve and right ventricle. In addition, abnormal reflux between the various arteries of such fetuses can also be detected. Postpartum children are assessed by measuring the size and function of the right ventricle, the size of the tricuspid valve, and the degree of reflux ${ }^{21,22}$.

\subsubsection{Physical examination and imaging evaluation}

Physical examination and imaging assessment were performed by two cardiothoracic surgeons as well as two imaging specialists in our hospital. The physical examination showed cyanosis, wheezing, and shortness of 
breath, and continuous murmurs could be caught on the precordium. And the relevant auxiliary examinations before the surgery including CT scan, electrocardiogram and X-ray of chest are presented inFigure 2 . After obtaining written consent and signature from all participants or their legal guardians, we take venous blood from six people in the family. The collection and use of patient biological samples were reviewed by the Shanghai Children's Hospital Ethics Committee.

\subsection{Whole exome sequencing and Sanger sequencing verification}

\subsubsection{Extraction of genetic DNA}

Genomic DNA extraction of $2 \mathrm{ml}$ peripheral blood samples were collected from 6 family members participating according to the manufacturer's experimental procedure (QIAamp DNA Blood Mini Kit). Analysis of DNA degradation and RNA contamination by agarose gel electrophoresis. Detection of DNA purity by spectrophotometer. Then detect DNA purity by spectrophotometer. Accurate quantification of DNA concentration. And finally record the purity and concentration of the samples and store them in the refrigerator at $-80{ }^{\circ} \mathrm{C}$.

\subsubsection{Methods of Whole Exome Sequencing and identification of the variants}

The SeqCap_EZ_ExomeV3 (Nimblegen) sequencing capture system was used in the capture of the entire exome sequencing coding region. The procedure of sequencing was done on HiSeqTM 2000, and the results

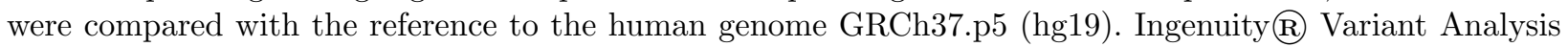
(QIGENE) was used in variational annotation filtering procedure. Sanger sequencing was performed on the 3500DX Genetic Analyzer (ABI). And the results comparisons and analysis were performed on Mutation Surveyor 4.0 (SoftGenetics) software.

\subsubsection{Sanger sequencing verification}

To identify pathogenic variants, we evaluated the pathogenicity of the 6 dominant variants using bioinformatics tools, such as PCR amplification and Sanger sequencing, and the mutation detection within the cases including2 patients with PA and 5 other PA-free relatives. And meanwhile verify that this mutation is co-segregating with the disease.

\subsubsection{Cytogenetic analysis}

After DNA extraction with the method in step 1.3.1,array comparative genomic hybridization (aCGH) experiments were performed using Agilent Human Genome CGH 4x180 oligonucleotide arrays (Agilent, Santa Clara, CA). These microarrays contain 180000 probes with a $13 \mathrm{~kb}$ average probe spacing. Arrays were scanned with the Agilent scanner and analysed with Cytogenomics 3.0.2.11 software (Agilent). The following databases were used for the analysis: DGV (http://dgv.tcag.ca), DECIPHER (https://decipher.sanger.ac.uk/), OMIM (https://www.omim.org), ClinGen (https://www.clinicalgenome.org/), Orphanet (https://www.orpha.net/consor/cgi-bin/index.php) and PubMed (https:// www.ncbi.nlm.nih.gov)

\section{Results}

In this study, 2 cases of PA patients and other family members without PA in the family were subjected to full exome sequencing analysis. Firstly, standard karyotype on these family members revealed a normal 46,XY karyotype. Then the array-CGH performed on the proband with PA did not show any chromosomal imbalance. The original sequencing sequences obtained by sequencing of each sample were finely filtered and then subjected to subsequent bioinformatics analysis. The target area coverage was $99.41 \%$, average 
coverage depth $>20 \mathrm{x}$. The whole exome capture was successful and the sequencing quality was reliable. Two cases of PA and the proband's mother who was considered to be a carrier of the mutated gene shared the mutant gene. And the variation that the proband's sister who is considered as a normal control does not have. Therefore, the mutant gene was considered as a candidate gene mutation. In order to refine our list of mutations, we finally screened for mutations that were predicted to be functionally harmful by SIFT and CADD, and which were not reported in any database. The score of CADD was 22.8. according to the guidelines for genetic variant interpretations $(\mathrm{ACMG})$ by Richards et al., the mutation was variant uncertain significance (VUS).But in this family, both the two children gained the disease, and the mother who carries the mutation develop cardiovascular diseases symptoms. Furthermore, only one mutation was found in this family associated with this disease. Our test identified a novel homozygous missense variant c.2804C $>$ T (p. A935V) in BMPR2.Moreover, the changes of construcions of the gene were proved to be associated with pulmonary diseases such as PAH, so in our opinion, the mutation was accosiated with the disease.

Whole exome sequencing results demonstrate that BMPR2 gene mutation may be related to the pathogenesis of PA. A heterozygous mutation in the BMPR2 gene c.2804C $>$ T (p. A935V) was rarely found in the normal database gnomAD (1/246048). Mutation of BMPR2 in the blood samples of the two cases of PA and the proband's mother who was considered to be a carrier of the mutated gene was found. And no mutation of BMPR2 in the blood samples of other family members in this family were treated as normal controls. The mutation was presented in Figure 3 .

\section{Discussion}

The PA disease is common complex cyanotic congenital heart disease. The etiology of PA disease is complex, and more and more studies have shown that PA is mainly related to genetic factors. In the present study, we performed whole exome sequencing on six members of the PA family to identify the disease-causing gene. Epidemiological studies have shown that the incidence of congenital heart disease in congenital malformations is $28 \%$, and the incidence of PA is $1.3 \%-3.4 \%$. In recent years, in addition to the classic MYH6, HAND1, HAND2, HEY family, etc., some new genes have been discovered. These genes control the recessive inheritance of congenital heart disease including CHD7, COL1A1, COL5A2, FBN2, NOTCH1, NSD1, and TSC2, as well as genes that only remain at animal level, such as CHD (DGCR2, DAW1, LRP1, and MYH10) and so $\mathrm{on}^{1}$. Bone morphogenetic protein (BMP) is a group of proteins that promote bone formation and act on cells such as osteoblasts, chondrocytes, and nerve cells that can affect embryonic growth and development ${ }^{23}$.BMP belongs to the multifunctional cytokine in the TGF-beta superfamily. When BMP2 binds to the cell surface threonine/serine kinase receptor, it can play a regulatory role. The TGF superfamily and related enzymes transmit signals through two types of heteropolypeptide receptors: BMPR1 (about 50-55 kD) and BMPR2 (about $70-80 \mathrm{kD}$ ).

In recent years, the genetic pathogenesis of PA has been confirmed by more and more studies. In 2014, Xie et al first discovered the relationship between copy number variation and $\mathrm{PA}^{5}$. These variations include $16 \mathrm{p} 13.1,22 \mathrm{q} 11.2,5 \mathrm{q} 14.1,10 \mathrm{p} 1$ and $17 \mathrm{p} 13.2^{5}$. There are four rare pathogenic mutations leading to PA, in which repeated mutations of DHFR5q14.1 and CUBN10p13 regulate folate-mediated metabolism, MTHFR regulates the metabolism of folic acid and vitamin B12, and 17q13.2 deletion regulates CAMT2 affects NKX2.5 ${ }^{24}$.In 2012 , Soemedi et al first proposed that the 1q21.1 mutation is a possible pathogenesis of congenital heart disease ${ }^{11}$. It was found by copy number variation analysis that most of the PA was caused by the GJA5 gene mutation. Similarly, the repetition of chromosomes such as 16p13.1, 5q14.1, 5q14.1, and $10 \mathrm{p} 13$ and the deletion of the $17 \mathrm{q} 13.2$ chromosome may also result in $\mathrm{PA}^{25}$. Single gene locus research, in recent years, found that GJA5, GJA1, GDF1, MTHFR, etc. ${ }^{7,26}$. may be related to the pathogenesis of PA. In 1995, Rosenzweig et al first discovered that transfected COS-1 cells, BMP7, and BMP4 (112262) were linked to BMPR $2^{27}$. And the chemical bonds between these connections are only stable in the presence of BMPR1. Most studies have found that mutations in the BMPR2 gene located on chromosome 2q33 are associated with the pathogenesis of most diseases, including familial pulmonary hypertension (PAH) and 
pulmonary veno-occlusive disease (PVOD) ${ }^{28}$. Certain deletions of the BMPR2 gene have been shown to trigger atherosclerosis in animal experiments ${ }^{29}$. Also included are some of the diseases caused by genes that interact with BMPR2: the Prader-Willi syndrome caused by the action of NIPA $1^{30}$.

In this study, we performed a complete exome sequencing, bioinformatics analysis and Sanger sequencing in a family of PA families inherited from three generations. It was found that the unreported new mutations in the BMPR2 gene may be related to the pathogenesis of PA in this family. This family is the genetic basis of the pathogenesis of PA, increasing the genetic profile of PA. It has been shown that BMPR2 prominently expressed in the vascular development and is associated with canonical WNT signaling pathway. And other than PA, the mutation could also lead to typical cardiovascular diseases such as PAH as well as obesity ${ }^{31}$. Both the proband and the proband's brother develop the disease, and the proband's mother who carrys the mutation of the gene also presents typical symptoms including obesity and other cardiovascular disease symptoms.

The study of these genes is either in the screening or screening stage, or in the animal experiment stage, but the correlation between them and the pathogenesis of PA is still unclear 3231303029 . This study is to verify the pathogenesis of PA by performing full exome sequencing and candidate mutation family members in a PA family member. In this study, we performed high-throughput second-generation sequencing technology for whole exome sequencing of patient samples. Whole exome sequencing uses target sequence capture technology to capture high-throughput DNA from all exome regions of the genome, which not only can quickly detect the pathogenic genes of rare genetic diseases, but also can be used for common diseases caused by multiple genes. Thereby revealing the genetic pathogenesis of these diseases.

In summary, the clinical manifestations, diagnostic criteria and treatment options of PA are relatively clear, and the concept that genetic factors play an important role in the pathogenesis of PA is also recognized by most clinicians and researchers. Gene mutations are associated with the pathogenesis of PA. But the pathogenesis of PA is still unclear. This study found in a PA family that BMP signaling pathway is possibly associated with the cardiac development and confirmed that mutations in this gene may be closely related to the pathogenesis of PA in this family. However, it is also necessary to verify the overall prevalence of mutations in the BMPR2 gene in the PA population in a large number of PA sporadic cases and PA families. Further in vivo and in vitro experiments are needed to determine whether the mutation does lead to the pathogenesis of PA and its pathogenesis, and elucidate the association between genotype-phenotype. At the same time, this study also suggests that the BMP family may be one of the important signaling molecules involved in the pathogenesis of PA. BMP signaling pathway may be one of the breakthroughs in the pathogenesis of PA.

\section{Concluding Remarks}

Here, we report a clinical case of rare PA in a Chinese family. This case highlights the importance of clinical diagnosis of the PA associated with mutation in BMPR2 gene. Hence, molecular genetic screening of BMPR2gene could be considered to possibly confirm the patients clinically diagnosed with PA. Our present study also emphasizes the significance of the high quality genetic analysis by whole exome sequencing in the molecular diagnosis of rare congenital heart diseases with possible phenotypic heterogeneity.

\section{Ethics Statement}

We obtained written informed consent for genomic analysis of the proband and his family members in accordance with the Declaration of Helsinki. The project was approved by the ethics committee of the Shanghai Children's Hospital and informed consent was obtained from all participants. The proband and his family members provided written informed consent for the publication of the proband's identifiable information. 


\section{Acknowledgements}

Our sincere thanks to the patient and his family members for their participation. This work was supported by Shanghai Children's Hospital.

\section{Author Contributions Statement}

MQ : drafting and perfect the manuscript; XL: genetic analysis ; LS, RZ ,JL and JG: patient workup. MQ, XL, LS, RZ, JL and JG: final approval for the version to be published and agreement to be accountable for all aspects of the work.

\section{Conflict of Interest Statement}

The authors declare that they have no competing interests

\section{Figures and tables}

TABLE 1:Table 1 presents the symptoms presented in this PA family. , /, and NA presents the member of this family prsents, not present or not known to them.

FIGURE 1 : The pedigree of three generations of the PA family.Pedigree of the three-generation, Chinese family with two affected individuals. Squares indicate males, and circles represent females. Black and white symbols represent affected and unaffected individuals, respectively. The proband (red arrow) is no longer alive (III-2").

FIGURE 2 (A-F): The relevant auxiliary examinations before the surgery: CT scan, electrocardiogram and X-ray of chest.

Figure 2A-2D are the CT scan before surgery (2A. the red arrow shows a narrow pulmonary artery of the proband; $2 \mathrm{~B}$. the red arrow shows the blood supply of the pulmonary artery was the descending aorta; 2C.D both show pulmonary atresia as well as an undeveloped pulmonary valve). Figure $2 \mathrm{E}$ is the electrocardiogram of the proband before surgery, which presents an atrial hypertrophy, ventricular hypertrophy, change in ST and $\mathrm{T}$ waves. Figure $2 \mathrm{~F}$ is the $\mathrm{X}$-ray of the chest of the proband before surgery, which presents an increased heart shadow. And the cardiothoracic ratio of the proband was about $75.9 \%$.

FIGURE 3(A-B) : A: A mutation was found in the proband, proband's mother as well as the proband's elder brother (Line 1,2 and 6). Line 1 to Line 6 represents for the proband, the proband's mother, the proband's father, the proband's sister, the proband's grandfather, the proband's grandmother and the proband's elder brother. B: The mutation is highly conserved in various species.

\section{References}

1. Gao M, He X, Zheng J. Advances in molecular genetics for pulmonary atresia. Cardiol Young. 2017;27(2):207-216.

2. Elias P, Poh CL, du Plessis K, et al. Long-term outcomes of single-ventricle palliation for pulmonary atresia with intact ventricular septum: Fontan survivors remain at risk of late myocardial ischaemia and death. Eur J Cardiothorac Surg.2018;53(6):1230-1236. 
3. Yang SH, Luo PH, Tian XX, et al. Prenatal diagnosis of pulmonary atresia with ventricular septal defect. $J$ Med Ultrason (2001).2018;45(2):341-344.

4. Gomez O, Martinez JM. Pulmonary Stenosis and Atresia. 2018:373-377.e371.

5. Xie L, Chen JL, Zhang WZ, et al. Rare de novo copy number variants in patients with congenital pulmonary atresia. PLoS One.2014;9(5):e96471.

6. Mercer-Rosa L, Elci OU, Pinto NM, Tanel RE, Goldmuntz E. 22q11.2 Deletion Status and Perioperative Outcomes for Tetralogy of Fallot with Pulmonary Atresia and Multiple Aortopulmonary Collateral Vessels.Pediatr Cardiol. 2018.

7. Guida V, Ferese R, Rocchetti M, et al. A variant in the carboxyl-terminus of connexin 40 alters GAP junctions and increases risk for tetralogy of Fallot. Eur J Hum Genet. 2013;21(1):69-75.

8. Sun X, Meng Y, You T, et al. Association of growth/differentiation factor 1 gene polymorphisms with the risk of congenital heart disease in the Chinese Han population. Molecular Biology Reports.2013;40(2):12911299 .

9. Capelli C, Sauvage E, Giusti G, et al. Patient-specific simulations for planning treatment in congenital heart disease. Interface Focus. 2018;8(1):20170021.

10. He X, Gao B, Shi G, et al. Surgical strategy and outcomes for the delayed diagnosis of pulmonary atresia with intact ventricular septum.J Cardiol. 2018.

11. Soemedi R, Topf A, Wilson IJ, et al. Phenotype-specific effect of chromosome 1q21.1 rearrangements and GJA5 duplications in 2436 congenital heart disease patients and 6760 controls. Hum Mol Genet. 2012;21(7):1513-1520.

12. Kozyraki R. Cubilin, a multifunctional epithelial receptor: an overview. Journal of Molecular MedicineJmm. 2001;79(4):161-167.

13. Tsaroucha AK, Chatzaki E, Lambropoulou M, et al. Megalin and cubilin in the human gallbladder epithelium. Clinical and Experimental Medicine. 2008;8(3):165-170.

14. Zhang DM, Mehler MF, Song QB, Kessler JA. Development of bone morphogenetic protein receptors in the nervous system and possible roles in regulating trkC expression. J Neurosci. 1998;18(9):3314-3326.

15. Kim M-J, Park SY, Chang HR, et al. Clinical significance linked to functional defects in bone morphogenetic protein type 2 receptor, BMPR2.BMB Reports. 2017;50(6):308-317.

16. Orriols M, Gomez-Puerto MC, Ten Dijke P. BMP type II receptor as a therapeutic target in pulmonary arterial hypertension. Cellular and molecular life sciences : CMLS. 2017;74(16):2979-2995.

17. Garcia-Rivas G, Jerjes-Sanchez C, Rodriguez D, Garcia-Pelaez J, Trevino V. A systematic review of genetic mutations in pulmonary arterial hypertension. BMC Med Genet. 2017;18(1):82.

18. Hardwick JC, Kodach LL, Offerhaus GJ, van den Brink GR. Bone morphogenetic protein signalling in colorectal cancer. Nature reviews Cancer. 2008;8(10):806-812.

19. Kim HH, Hyung WJ, Cho GS, et al. Morbidity and mortality of laparoscopic gastrectomy versus open gastrectomy for gastric cancer: an interim report-a phase III multicenter, prospective, randomized Trial (KLASS Trial). Annals of surgery. 2010;251(3):417-420.

20. Finkenzeller G, Hager S, Stark GB. Effects of bone morphogenetic protein 2 on human umbilical vein endothelial cells. Microvascular Research. 2012;84(1):81-85.

21. Todros T, Paladini D, Chiappa E, et al. Pulmonary stenosis and atresia with intact ventricular septum during prenatal life. Ultrasound in Obstetrics \&3 Gynecology. 2003;21(3):228-233. 
22. Maeno YV, Boutin C, Hornberger LK, et al. Prenatal diagnosis of right ventricular outflow tract obstruction with intact ventricular septum, and detection of ventriculocoronary connections. Heart.1999;81(6):661668.

23. Zhao L, Zhou Z, Wang S, et al. A recurrent mutation in bone morphogenetic proteins-2-inducible kinase gene is associated with developmental dysplasia of the hip. Exp Ther Med.2017;13(5):1773-1778.

24. Song K, Backs J, McAnally J, et al. The transcriptional coactivator CAMTA2 stimulates cardiac growth by opposing class II histone deacetylases. Cell. 2006;125(3):453-466.

25. Tomita-Mitchell A, Mahnke DK, Struble CA, et al. Human gene copy number spectra analysis in congenital heart malformations. Physiological Genomics. 2012;44(9):518-541.

26. Izumi K, Lippa AM, Wilkens A, Feret HA, McDonald-McGinn DM, Zackai EH. Congenital heart defects in oculodentodigital dysplasia: Report of two cases. American Journal of Medical Genetics Part A.2013;161(12):3150-3154.

27. Rosenzweig BL, Imamura T, Okadome T, et al. Cloning and characterization of a human type II receptor for bone morphogenetic proteins. Proceedings of the National Academy of Sciences.1995;92:7632-7636.

28. Girerd B, Lau E, Montani D, Humbert M. Genetics of pulmonary hypertension in the clinic. Current opinion in pulmonary medicine. 2017;23(5):386-391.

29. Lu H, Daugherty A. Atherosclerosis. Arteriosclerosis, thrombosis, and vascular biology. 2015;35(3):485491.

30. Tsang HTH, Edwards TL, Wang X, et al. The hereditary spastic paraplegia proteins NIPA1, spastin and spartin are inhibitors of mammalian BMP signalling. Human Molecular Genetics.2009;18(20):3805-3821.

31. Schulz TJ, Tseng YH. Emerging role of bone morphogenetic proteins in adipogenesis and energy metabolism. Cytokine 83 Growth Factor Reviews. 2009;20(5-6):523-531.

32. Contribution of rare inherited and de novo variants in 2,871 congenital heart disease probands.

Hosted file

TABLE 1.pdf available at https://authorea.com/users/375564/articles/492750-whole-exomesequencing-identifies-a-novel-pathogenic-bmpr2-variant-in-pulmonary-atresia 


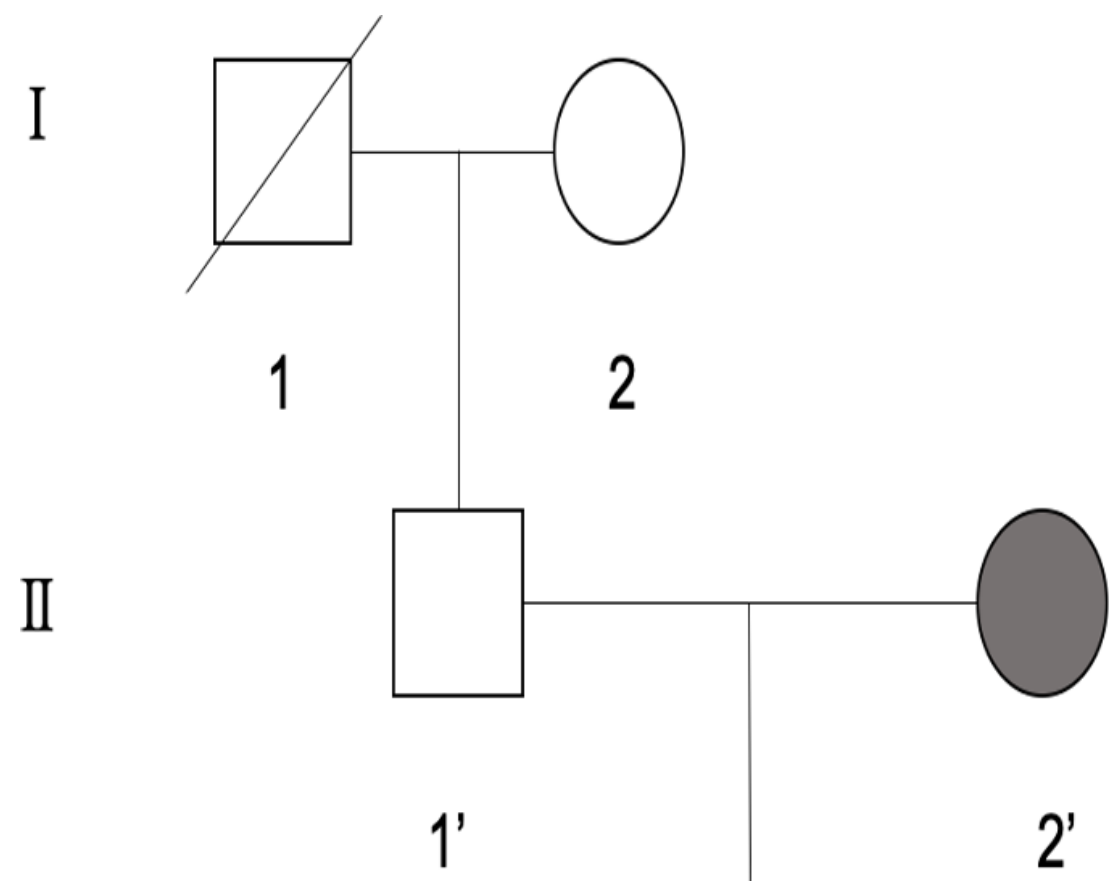

III

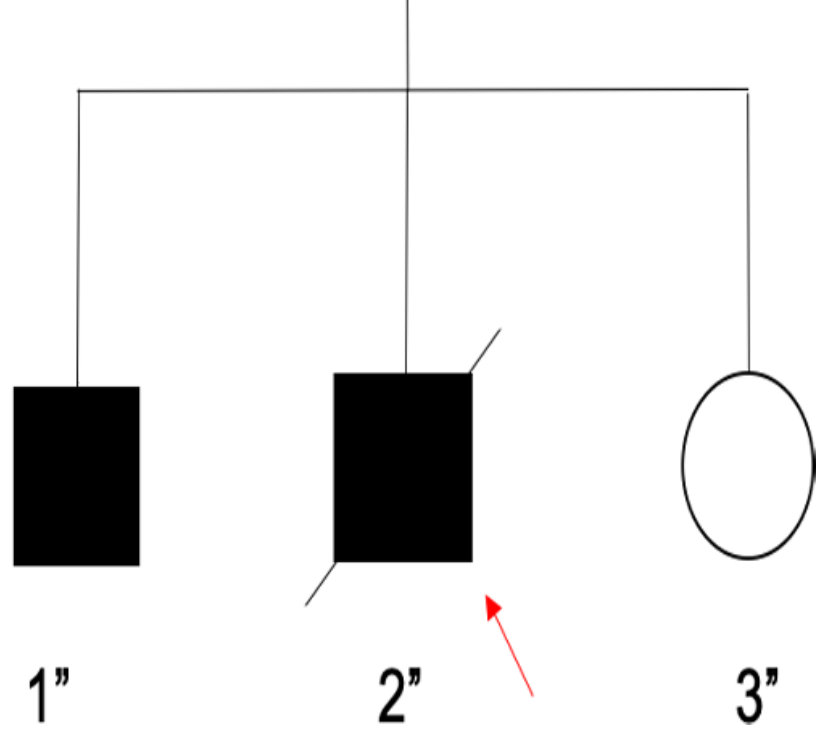


A

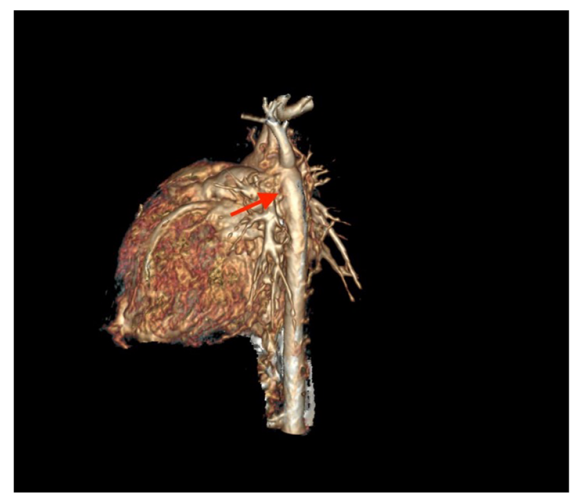

C

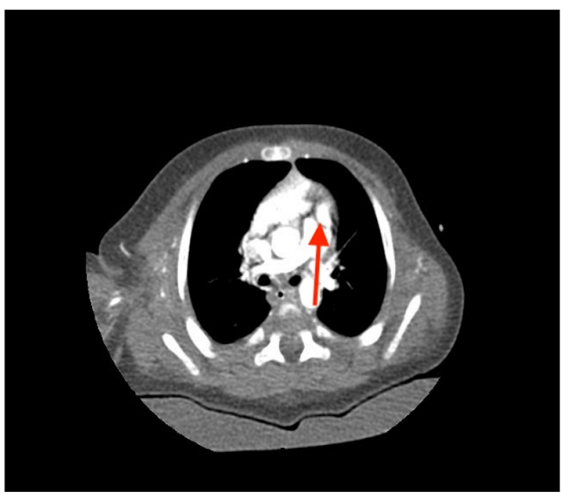

E

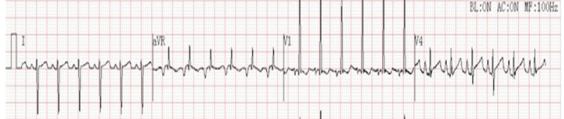

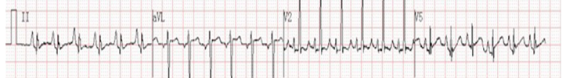

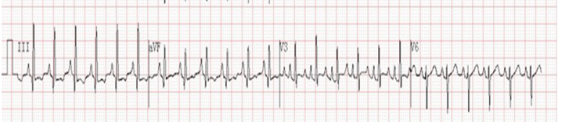

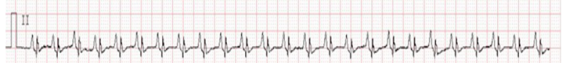

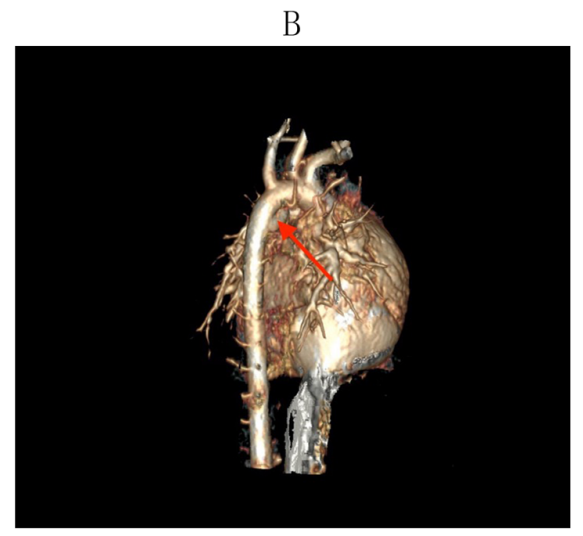

D

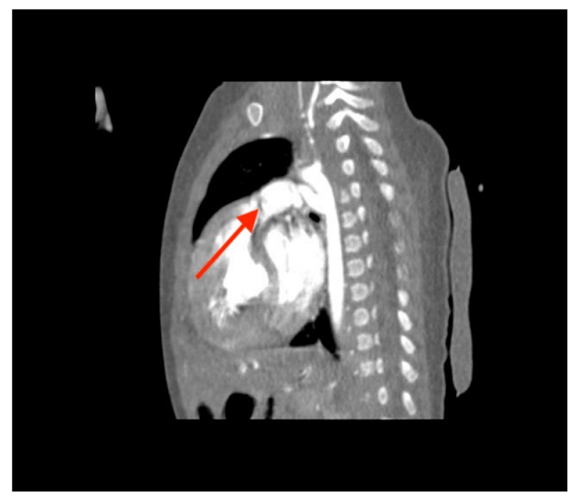

F

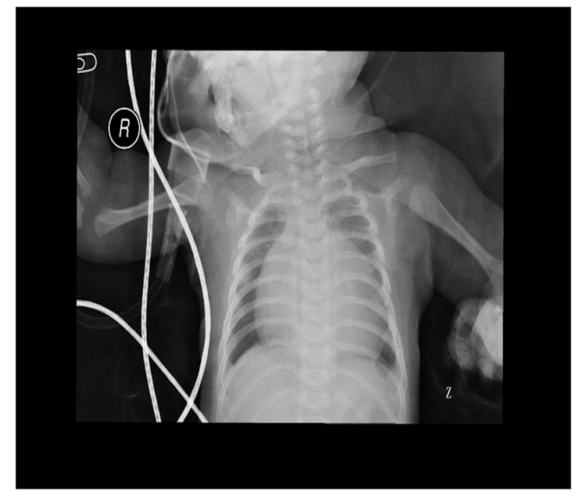


A

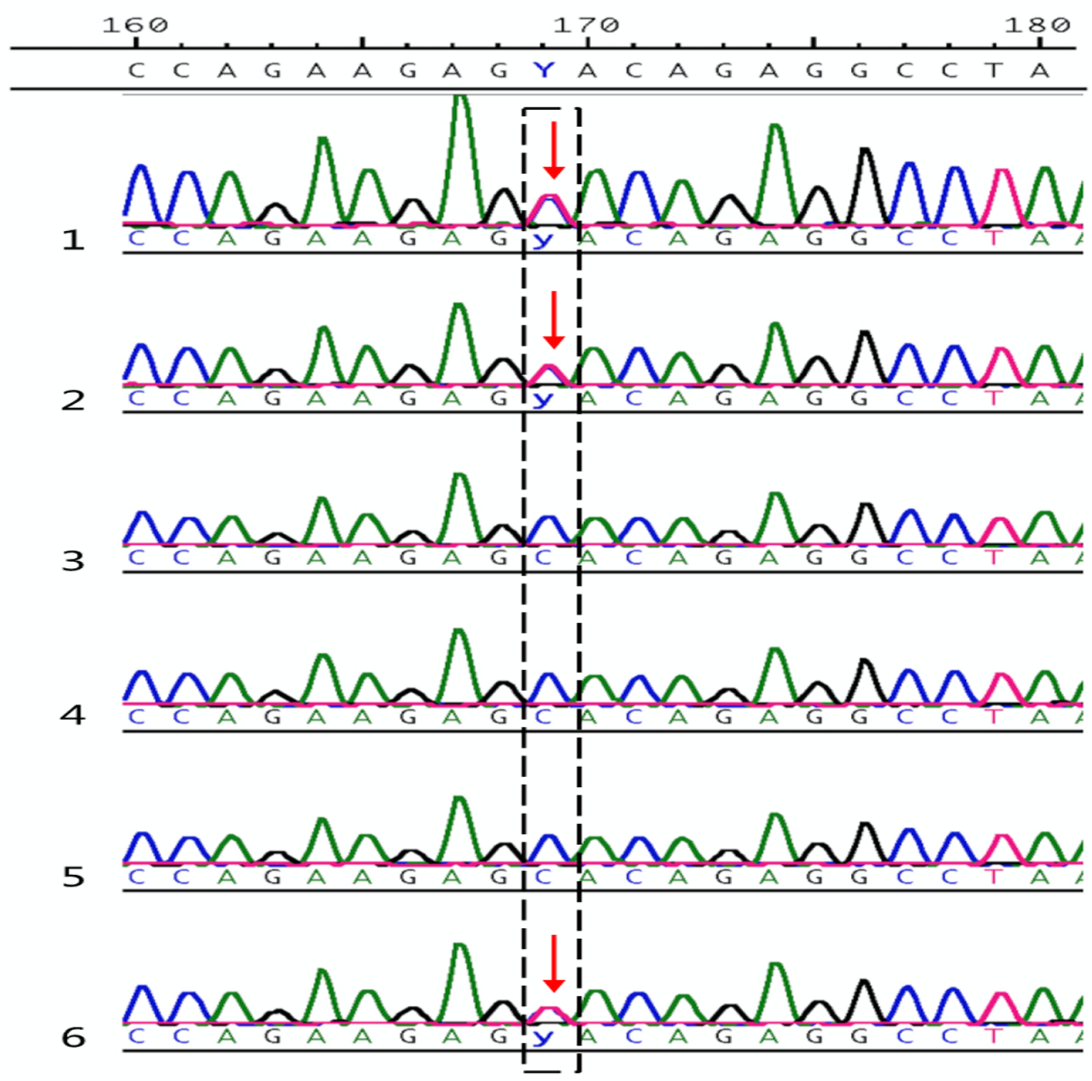

B

$\begin{array}{ll}\text { Human } & \text { TAADPGPSKPRRAQRPNSLDLSATN } \\ \text { Rhesus } & \text { TVADPGPSKPRRAQRPNSLDLSATN } \\ \text { Mouse } & \text { TAADPGPSKPRRAQRPNSLDLSATN } \\ \text { Rabbit } & \text { TVADPGPSKPRRAQRPNSLDLSATN } \\ \text { Pig } & \text { TVADPAPSKPRRAQRPNSLDLSATN } \\ \text { Dog } & \text { TVADPGPSKPRKAQRPNSLDLSATN } \\ \text { Elephant } & \text { TVPDPGPSKPRRAQRPNSLDLSATN } \\ \text { Chicken } & \text { VARNPGQTQTRRAQRPNSLDLSATN }\end{array}$

